\title{
Application of frictional heat signatures for prediction of structural vibrational characteristics
}

\author{
S. M. Talai ${ }^{1}$, D. A. Desai ${ }^{1}$ \& P. S. Heyns ${ }^{2}$ \\ ${ }^{I}$ Department of Mechanical Engineering, Mechatronics and \\ Industrial Design, Tshwane University of Technology, South Africa \\ ${ }^{2}$ Centre for Asset Integrity Management, Department of Mechanical and \\ Aeronautical Engineering, University of Pretoria, South Africa
}

\begin{abstract}
Frictional damping of vibrating components such as turbomachinery blades, generally result in a temperature increase at the blade-lacing wire interface due to blade harmonic motion during operation. Interestingly, studies have shown that temperature is amongst the most common indicators of structural health of a dynamic component. Nevertheless, its application to measurement of vibration characteristics has not yet been contemplated and thus relatively unique. Hence, the aim of this work is to develop a novel technique for acquiring structural vibrational characteristics from frictional heat signatures. To facilitate this, an experimentally validated finite element model (FEM) was developed. The skillful manipulation of heat conduction equations and a finite difference approach; successfully identified the vibration behavior. The implications and benefits of the developed technique for structural integrity and condition monitoring applications are numerous, for example; the early detection of structural defects. Furthermore, this approach has great potential of overcoming most of the limitations experienced with conventional methods such as strain gauging which encounter fatigue during vibration measurement and may thus compromise measurements. Keywords: infrared thermography, frictional heat signature, vibration, finite element modelling.
\end{abstract}




\section{Introduction}

Generally, friction is considered by engineers as detrimental to the design of mechanisms with mating parts in relative motion [1]. However, it has long been established that it can as well provide a very effective means of damping out vibrations in elastic structures such as low pressure (LP) steam turbine blades [2, 3]. Subsequently, lacing wires are incorporated for enhancing passive damping, fig. 1. These dampers dissipate the vibratory energy that ultimately leads to temperature increase at the contact interface [2]. Harrison [4] observed that where there is heat, probably there is vibration.

Even though temperature is amongst the most common indicators of structural health of dynamic components [5]; it is unfortunate that no studies have been carried out exploiting the frictional heat signature for the prediction of vibrational characteristics. Therefore, it is the aim of this study to develop a technique that can facilitate the prediction and monitoring of vibration behaviour from frictional heat signatures. This has been inspired by the fact that, infrared thermography has matured and widely accepted as a condition monitoring tool where temperature is measured in a non-contact manner [5].

Furthermore, it is worth mentioning that numerical approaches to the simulation of the vibration characteristics for dynamic structures has become popular in recent years [6]. Jianzhong et al. [7] state it is the most powerful technique for solving complex mechanical structural vibration problems. Also, it is important to point out that FEM is a dominant tool for the effective understanding of the behaviour governing turbomachinery blade excitation to its heat generation at the blade-lacing wire interface in relation to the structural vibration frequency. The FEM has a great advantage of performing the multi blade excitation and thermal data extraction with high accuracy that is otherwise difficult to be achieved through experimentation. Above all, experimentation is generally expensive compared to FEM simulations. In the light of the above, this work employs an experimentally validated FEM of a simplified turbomachinery blade with a single continuous lacing wire subjected to sinusoidal loading for the purpose of achieving the above aim.

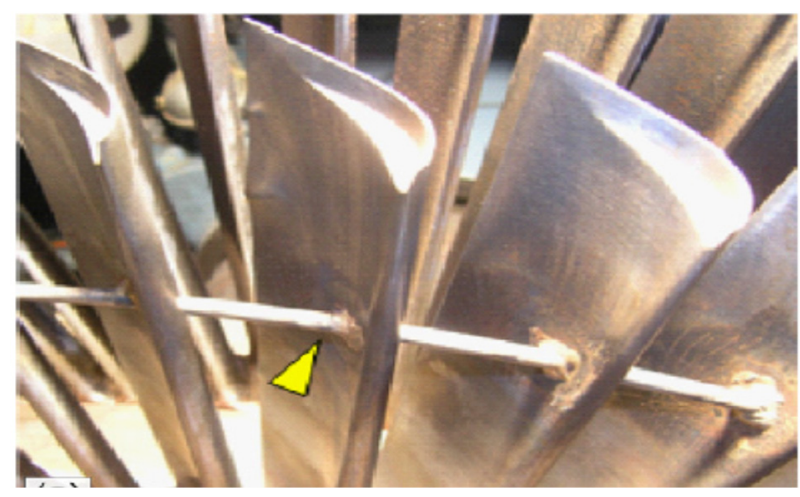

Figure 1: LP steam turbine blade with single continuous lacing wire [8]. 


\section{Mathematical model}

Modelling of frictional heat for actual turbomachinery such as for LP steam turbine blades with lacing wire is very complicated [3]. However, since the objective of this work is to develop a methodology for acquiring structural vibration characteristics from thermal signatures; a simplified structure composing of two rectangular blades and a friction damper (lacing wire) were adopted.

\subsection{Formulation of the loading conditions}

The blade loading due to perturbation can be expressed as

$$
F(\omega, t)=F_{n} \operatorname{Sin}(\omega t)
$$

where $F(\omega, t)$ is a varying force function, $F_{n}$ is the normal excitation force at a point in time $(t)$ at circular frequency $\omega$. Furthermore, centrifugal force inherent to the friction damper can be represented by

$$
F_{c}=m r \omega^{2}
$$

where $F_{c}$ is the centrifugal force, $m$ is the friction damper mass, $r$ is the location and $\omega$ is the angular velocity. Consequently, the structural excitation frequency, $f_{e x}$ from the periodic time, $T_{p}$ of oscillation is given by

$$
f_{e x}=\frac{1}{T_{p}}
$$

and the structural displacement, $u$ from measured velocity response is given by

$$
u=v \times t
$$

where $u$ is the displacement, $v$ is the velocity at time t.

The geometric dimensions and material properties used in this study are given in tables 1 and 2 , respectively.

Table 1: Geometric dimensions.

\begin{tabular}{|l|c|}
\hline Description & Dimension \\
\hline Blade mass, $m$ & $0.10 \mathrm{~kg}$ \\
\hline Length, $L$ & $300 \mathrm{~mm}$ \\
\hline Width & $25 \mathrm{~mm}$ \\
\hline Thickness & $2 \mathrm{~mm}$ \\
\hline Wire diameter & $5 \mathrm{~mm}$ \\
\hline Blade hole diameter [9], [grade: $F 8 / j 7$ ] & $5_{+0.412}^{+0.42} \mathrm{~mm}$ \\
\hline Location of blade damper hole from blade root, $L_{d}$ & $250 \mathrm{~mm}$ \\
\hline Location of the exciter, $L_{e x}$ & $290 \mathrm{~mm}$ \\
\hline
\end{tabular}


Table 2: $\quad$ Material properties [10].

\begin{tabular}{|l|c|}
\hline Structural material properties & $7740 \mathrm{~kg} / \mathrm{m}^{3}$ \\
\hline Density, $\rho$ & $200 \mathrm{GPa}$ \\
\hline Young modulus, $E$ & 0.33 \\
\hline Poisson ratio, $v$ & 0.15 \\
\hline Static friction coefficient $[11], \mu_{s}\left(\mu_{k}=0.75 \mu_{s}\right)$ & $16.5 \mathrm{~W} / \mathrm{m}^{2} . K$ \\
\hline Thermal material properties & $500 \mathrm{~J} / \mathrm{kg} . \mathrm{K}$ \\
\hline Thermal conductivity, $k$ &
\end{tabular}

\subsection{Frictional heat modelling}

The interface frictional heat generation, $q_{g}$ due to relative motion is given by

$$
q_{g}=\mu \times u F_{n}
$$

where $\mu$ is the coefficient of friction, $u$ is the interface relative velocity and $F_{n}$ is the normal force exciting the structure. The heat conduction equation in the Cartesian co-ordinate system $(x, y, z)$ for a differential element is expressed as [12]

$$
\frac{\partial^{2} \theta}{\partial x^{2}}+\frac{\partial^{2} \theta}{\partial y^{2}}+\frac{\partial^{2} \theta}{\partial z^{2}}+\frac{q g}{k}=\frac{1}{\alpha} \frac{\partial \theta}{\partial t}
$$

where $\theta$ is the temperature rise on the blade surface, $k$ is the thermal conductivity, $\rho$ is the material density, $c$ is the specific heat capacity, $q_{g}$ is the heat generated per unit volume and $\alpha$ is the thermal diffusivity. The grid applied (in relation to the above co-ordinate system) to the blade surface can be utilised for the analysis of heat distribution, fig. 2 . Thus, the temperature rise equations in $\Delta x$ and $\Delta y$ coordinates are

$$
\frac{\partial^{2} \theta}{\partial x^{2}}=\frac{1}{(\Delta x)^{2}}\left(\theta_{i+1}-2 \theta_{i, j}+\theta_{i-1}\right), \frac{\partial^{2} \theta}{\partial y^{2}}=\frac{1}{(\Delta y)^{2}}\left(\theta_{j+1}-2 \theta_{i, j}+\theta_{j-1}\right)
$$

Assuming that the heat has reached steady state condition and that the convectional heat loss on both sides obey Newton's law of cooling [13]; eqn. (6) reduces to

$$
q_{g}(x, y)=k\left[\frac{2 h_{c o n}}{c k} \theta_{i, j}-\frac{\partial^{2} \theta}{\partial x^{2}}-\frac{\partial^{2} \theta}{\partial y^{2}}\right]
$$

The steady state displacement response due to sinusoidal loading is given by [14] as

$$
y(t)=Y \sin (\omega t-\phi)
$$

where $Y$ is the amplitude, $\omega$ is the circular frequency and $\phi$ is the phase angle. 


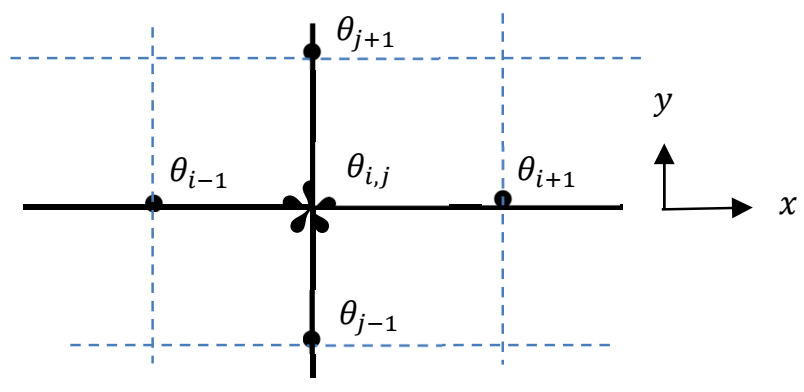

Figure 2: Temperature distribution grid.

Consequently, the frictional heat generation, $q_{g}$ per half a cycle is of the form $2 \mu N f Y$ [13]; hence

$$
q_{g}=\frac{2 \mu N f y(t)}{\operatorname{Sin}(\omega t-\phi)}
$$

Therefore, manipulating eqns (7) and (10); the correlation for computing structural displacement from frictional heat signature can be developed as

$$
y(t)=\frac{k \sin (\omega t-\phi)}{2 \mu N \omega}\left[\frac{2 h_{c o n}}{c k} \theta_{i, j}-\frac{\partial^{2} \theta}{\partial x^{2}}-\frac{\partial^{2} \theta}{\partial y^{2}}\right]
$$

\section{Finite element modelling}

The following assumption were made during the FEM formulation:

- The radiation and heat losses are negligible in comparison to conduction and convection [13].

- The thermal conductance governs the interface properties.

- The frictional heat transfer obeys the heat partition model [15].

$$
r=\frac{k_{b}}{k_{b}+k_{d}}
$$

where $k_{b}$ and $k_{d}$ are the thermal conductivity of blade and damper respectively.

The FEM model composed of 3D deformable solid parts; these are two rectangular blades and a round bar acting as a friction damper (fig. 3). Finite element models of healthy and defect introduced (induced cuts of $6 \mathrm{~mm}$ and $8 \mathrm{~mm}$ deep) blades were developed. Encastre boundary conditions were defined for both blades while asymmetric boundary conditions were applied at the centre of the friction damper. The interface property was model with tangential frictional behaviour in accordance to heat partition ratio, thermal conductance and heat generation obeying the heat partition model described above. 


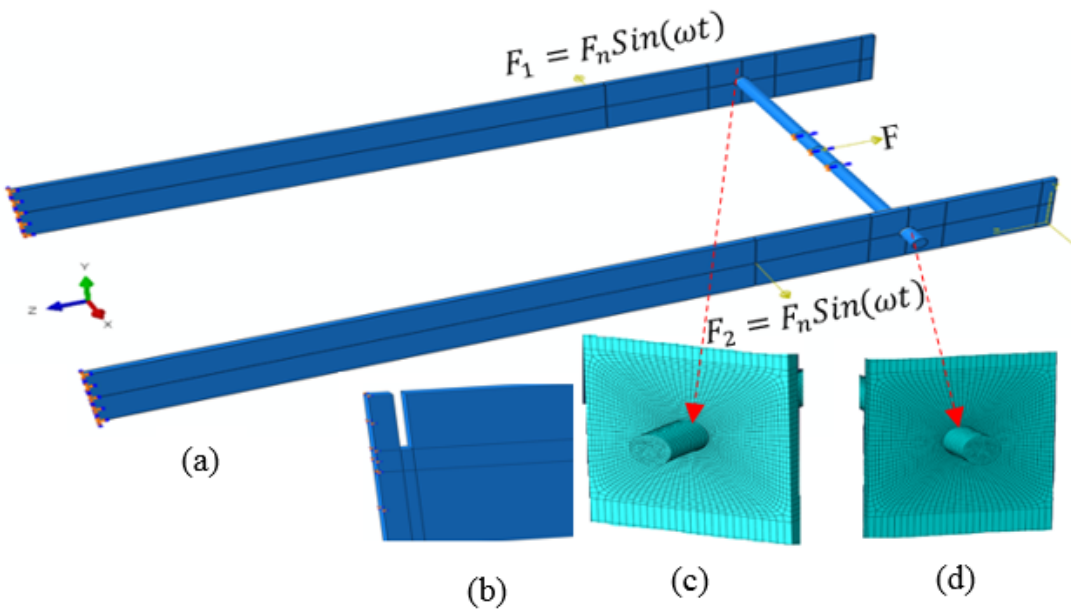

Figure 3: $\quad$ FEM model (a) assembly, B.C and loading (b) blade defect-1 mm wide (c) blade 1 interface mesh with reference node (d) blade 2 interface mesh with reference node.

Consequently; the modelled blades were subjected to varying force function with circular frequency of $25 \mathrm{~Hz}$ and $50 \mathrm{~Hz}$; each with a normal force of $0.084 \mathrm{~N}$ (fig. 3). On the other hand, the damper was laterally loaded with $0.001 \mathrm{~N}$ to mimic the effects of centrifugal force. These loading parameters were computed based on the work Booysen et al. [16] on fatigue assessment of low pressure steam turbine blades. The heat losses due to convection were modelled using convection coefficient of $30 \mathrm{~W} / \mathrm{m}^{2} . \mathrm{K}$ at $22^{\circ} \mathrm{C}$ defined as a predefined field [15]. Coupled temperature-displacement transient analysis was employed as the modelling methodology. The blades and damper were meshed C3D8RT elements. Appropriate mesh sizes were used guided by mesh convergence study. For example, a total of 175,912 elements and 288,685 nodes were generated for the healthy blade FEM. Mesh density was increased in critical areas such as contact zones, in order to provide adequate contact compliance, increase the accuracy of the solution and speed up convergence. Reference nodes were created at each blade interface where temperature and displacement time histories were required.

\section{Experimental setup}

The frictionally damped healthy cantilever blade was considered for the partial validation of FEM simulation results, fig. 4 in terms of frequency content. The lateral force of $2.25 \mathrm{~N}$ measured using a PCB force transducer model $208 \mathrm{C} 02$ with sensitivity rating of $11.24 \mathrm{mV} / \mathrm{N}$ was applied to the friction damper to mimic the effect of centrifugal force. 

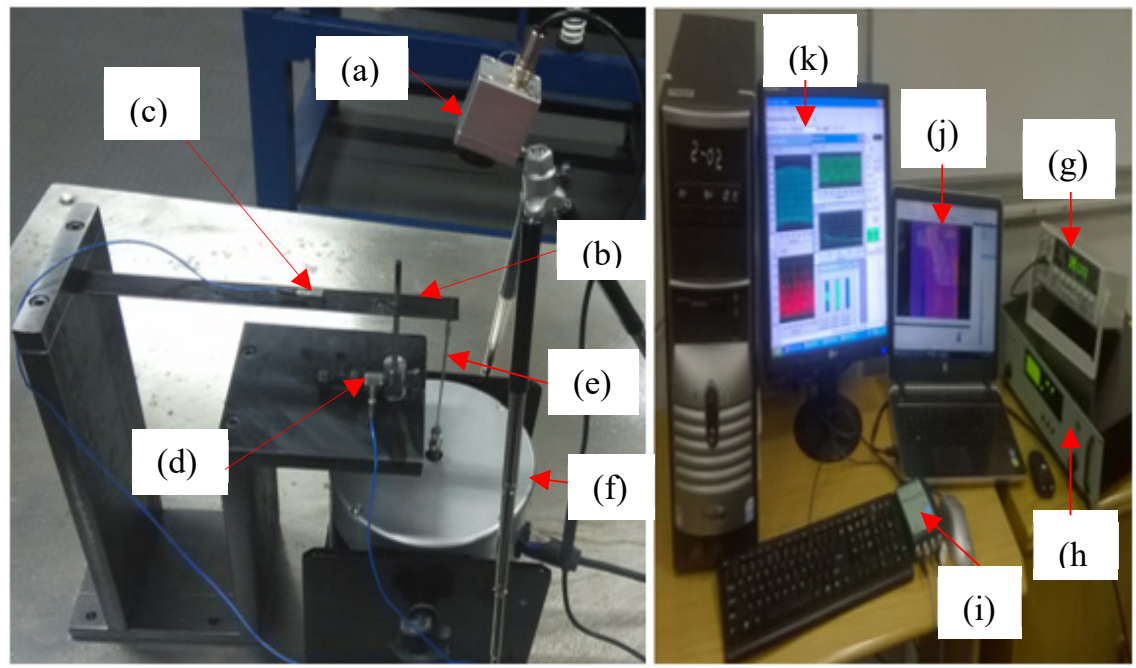

Figure 4: Frictional thermal analysis experimental set-up (a) The infrared camera; (b) cantilever blade with a dry friction damper; (c) accelerometer; (d) force sensor; (e) stinger (f) exciter; (g) function generator; (h) Amplifier; (i) RT Pro Photon analyzer; (j) PC for IRT analysis and (k) PC for dynamic analysis.

The excitation frequency from the function generator (model FG-7005C) was amplified (power amplifier model: LA-1500) before being transmitted to the modal exciter (Type: 4824, Bruel \& Kjaer); to facilitate blade excitation via a stinger. The blade displacement response was acquired using a miniature Deltatron accelerometer type 4507 with the sensitivity rating of $97.96 \mathrm{mV} / \mathrm{g}$ and mass of $4.8 \mathrm{~g}$ (Bruel \& Kjaer) that was mounted to the blade surface using loctite type 242. Concurrently, a micro-epsilon infrared camera with thermal sensitivity of $80 \mathrm{mK}$, lens of $23^{\circ}$ field of view, optical resolution of $120 \times 160$ pixels and real time thermography of $120 \mathrm{~Hz}$ frame rate was focused to the interface to capture thermal images. The problem of low thermal emissivity of the blade surface was eliminated by applying black paint. The room temperature during the experimentation was controlled at $23^{\circ} \mathrm{C}$.

\section{Results and discussion}

The temperature signature from the FEM simulation showed that there exists a threshold upon which the frictional heat is generated which is in agreement with that of Koh et al. [17] on characterization of turbine blade friction dampers. The phenomenon can be attributed to microslip. Furthermore, the results revealed that when blades are excited in phase; the temperature time history will be out of phase and vice versa (figs 5 and 6 ). Furthermore, it was observed that heat generation is 
dependent on the excitation frequency i.e. the higher the excitation frequency, the higher the rate of heat generation (figs 5-8). Consequently, the effect of structural defects resulted in an increase in heat generation which becomes more pronounced with increases in defect size (fig. 9).

Interestingly, the frictional temperature time histories were observed to be periodic, with well posed frequencies. Although the presence of defects did not present any effect on frequency from the temperature signature considering that this was a forced vibration, a significant increase of displacement was observed with $2.8 \%$ and $4.4 \%$ for defect depth size of $6 \mathrm{~mm}$ and $8 \mathrm{~mm}$, respectively (fig. 9).

The thermal radiometric data for frictional temperature signature corresponding to the hottest point (fig. 10); were analyst (figs 11 and 12). The signature indicated that it is periodic in nature with an average time of $0.02 \mathrm{~s}$ (fig. 12). Thus, using eqn (3); the structural excitation frequency from the captured frictional temperature signature was evaluated to be $50 \mathrm{~Hz}$ which correlates to the developed FEM results. In addition, the structural displacement was obtained from the developed correlation eqn (11) and interface temperature distribution data, fig. 10 and was computed to be $1.9 \mathrm{~mm}$.

However, the measured displacement velocity response using the described accelerometer, fig. 13; indicated that the time taken to attain the magnitude of $292.8367 \mathrm{~mm} / \mathrm{s}$ was $0.00684 \mathrm{~s}$. Hence, from eqn. (4); the structural displacement equates to $2.0 \mathrm{~mm}$. The relative difference being $5 \%$ with that of thermally predicted value. This can be attributed to the heat losses through convection and heat absorbed by both the blade and lacing wire during the excitation.

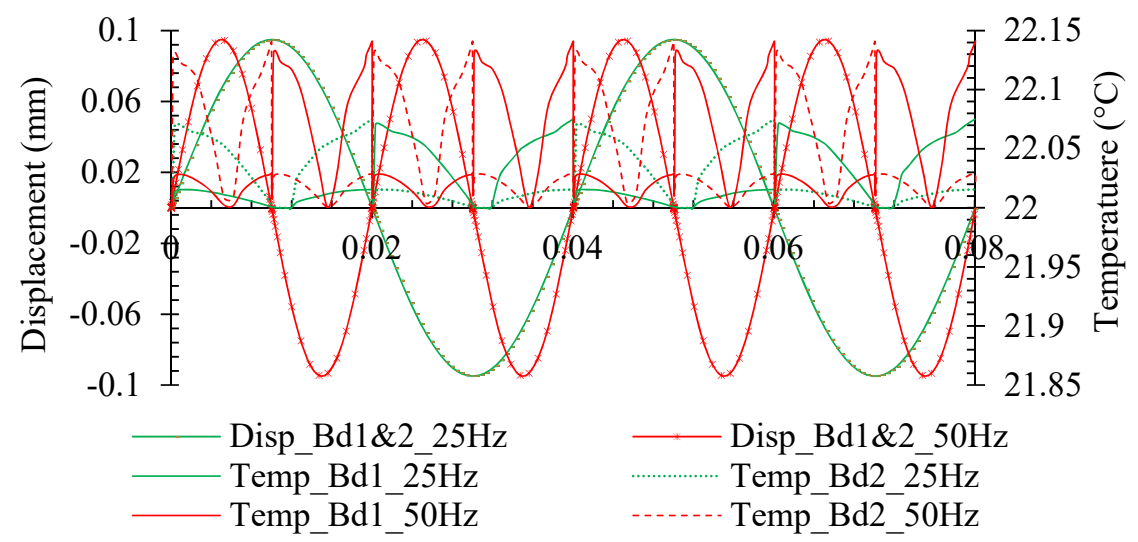

Figure 5: Temperature and displacement time history for in phase blades excitation at the same value of frequencies. 


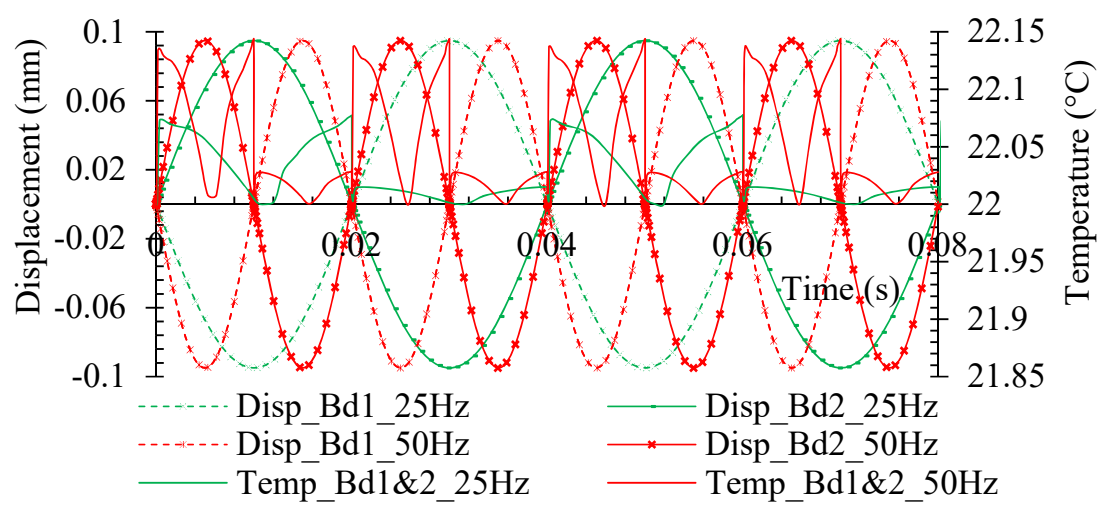

Figure 6: Displacement and temperature time history for out of phase blades excitation at same value of frequencies.

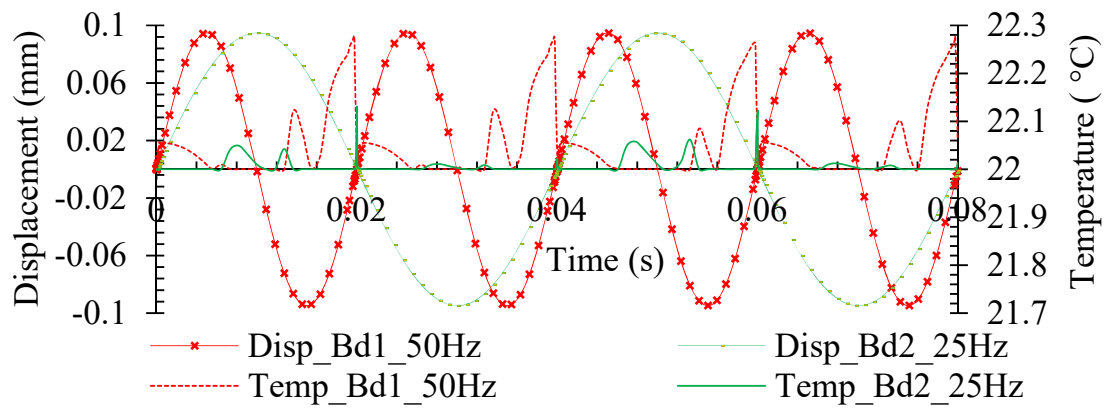

Figure 7: Displacement and temperature time history for in phase blades excitation at different value of frequencies.

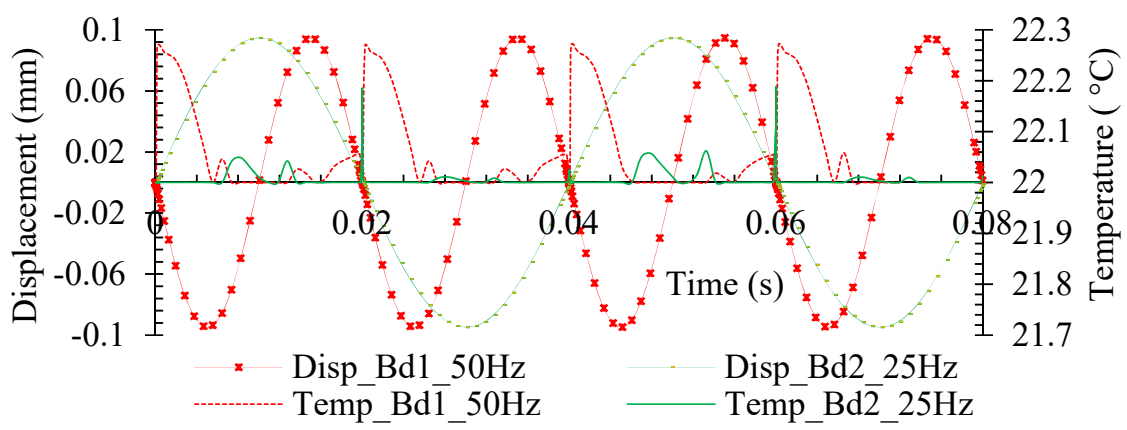

Figure 8: Displacement and temperature time history for out of phase blades excitation at different value of frequencies. 


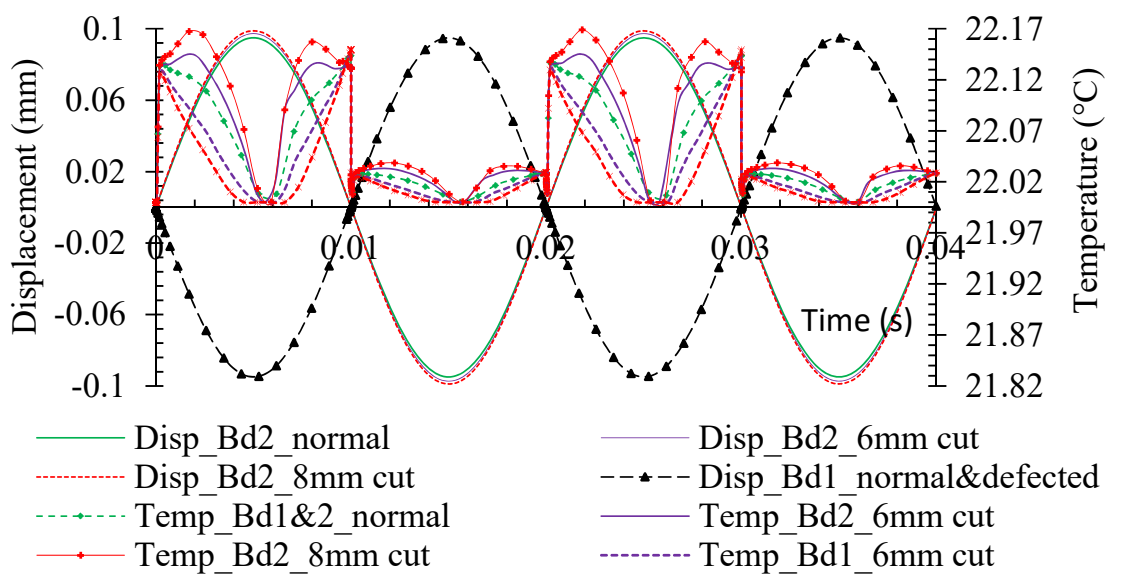

Figure 9: Effect of the structural defect.

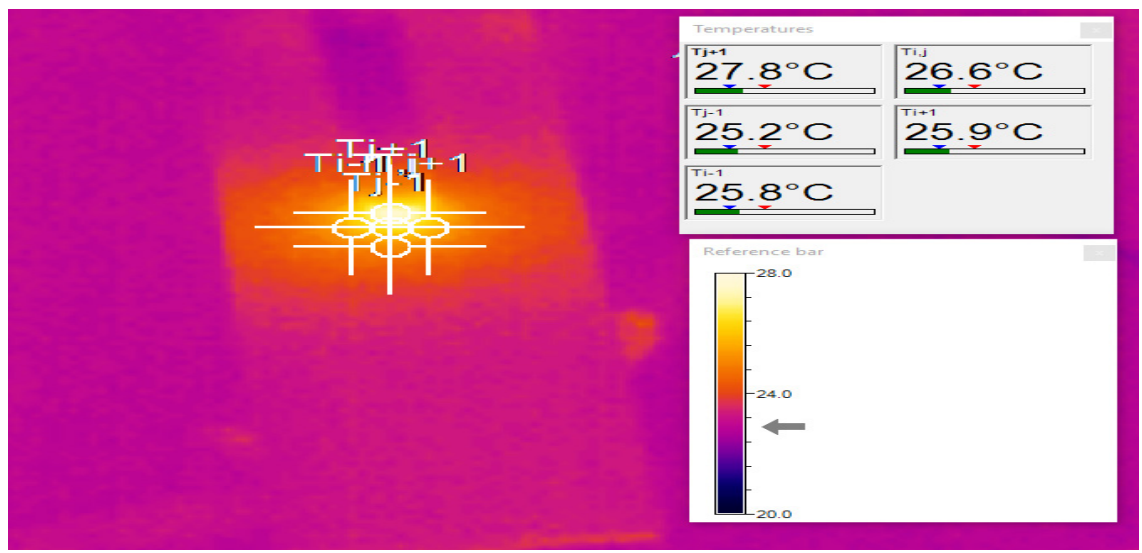

Figure 10: Frictional heat from IR thermal imager at the interface.

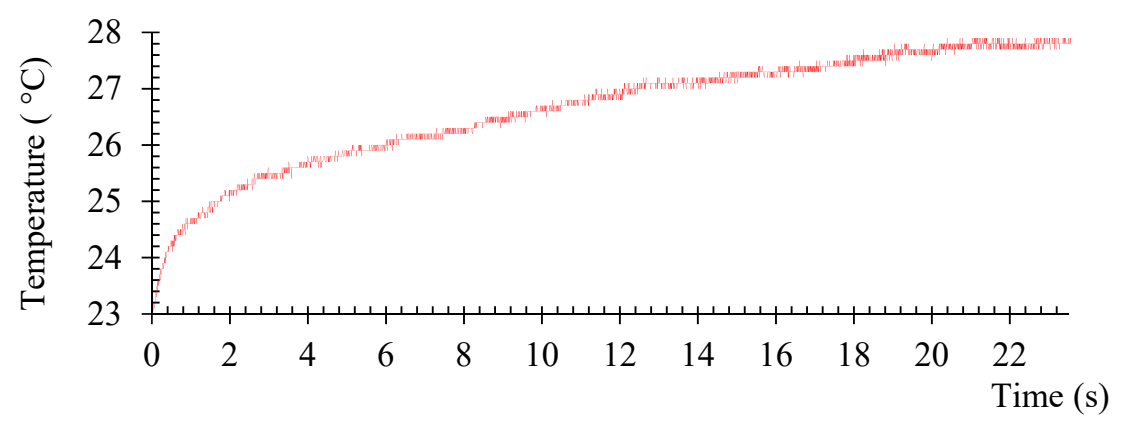

Figure 11: Interface temperature signature. 


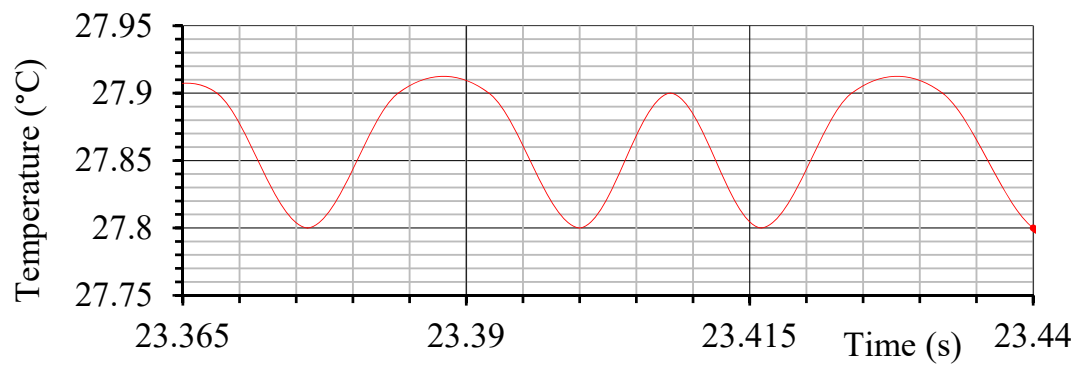

Figure 12: Small time interface temperature signature.

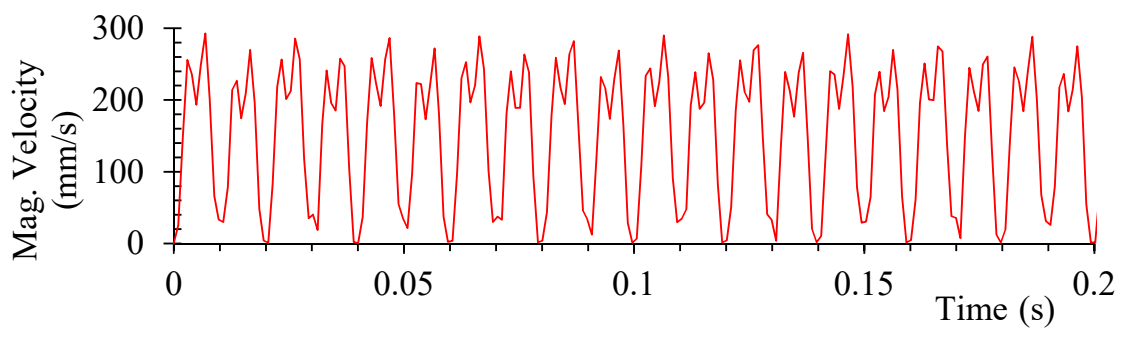

Figure 13: Blade displacement response.

\section{Conclusion}

The frictional temperature signature revealed that it follows Coulomb's law of friction and periodic in trend due to structural excitation. The FEM simulation results indicated frequencies for in phase excitation as $25.67 \mathrm{~Hz}$ and $50.78 \mathrm{~Hz}$ and out of phase excitation as $25.64 \mathrm{~Hz}$ and $50.35 \mathrm{~Hz}$. Interestingly, manipulation of the experimental temperature signature for blade excitation at $50 \mathrm{~Hz}$ showed good correlation to these values of excitation frequency while its displacement was $5 \%$ more than the measured value. This was attributed to the fact that the accelerometer was mounted towards the fixed of the cantilever blade. It can be seen that both the developed FEM and experimentally acquired frequency for the corresponding excitation are in good agreement with a relative difference of $0.81 \%$. Hence the FEM model is validated. In conclusion, the results of this study has successfully proved the capability of predicting the structural vibration characteristics from the frictional temperature signatures. This can form the basis for online structural integrity monitoring using temperature employing infrared thermography.

\section{Acknowledgements}

The authors greatly appreciate the support of Eskom Power Plant Institute (Republic of South Africa), University of Pretoria and Tshwane University of Technology for funding this research. 


\section{References}

[1] Straffelini, G., Friction and Wear: Methologies for design and control, Springer: Trento: 21-58, 2015.

[2] Singh, M. \& Lucas, G., Blade design \& analysis for steam turbines, The McGraw Hill Companies: United States of America: 20-60, 2011.

[3] Rao, S.J., Turbo-machine blade vibration (first edition), New Delhi: New age international publishers 7-20, 1991.

[4] Harrison, L.R., Shake, Rattle \& Roll: Where's there's Heat there's Probably Vibration. Sound and vibration, 37(5): 8-9, 2003.

[5] Bagavathiappan, S., Lahiri, B. B., Saravanan, T., Philip, J. \& Jayakumar, T., Infrared thermography for condition monitoring - A review. Infrared Physics \& Technology, 60: 35-55, 2013.

[6] Kadarno, P., et al., Vibration analysis of defected ball bearing using finite element model simulation. Proc. of the 9th conf. of Asian Pasific Industrial Engineering \& Management Systems. Nusa Dua, Bali: Indonesia: 28322840, 2008.

[7] Jianzhong, L., Seng, T.L., \& Zhangzhi, C., Numerical analysis of dynamic behavior of stream turbine blade group. Finite Elements in Analysis and Design, 35: 337-348, 2000.

[8] Poursaeidi, E. \& Arhani, M.R.M., Failure investigation of an auxiliary steam turbine. Engineering Failure Analysis, 17(6): 1328-1336, 2010.

[9] Sanders, W.P., Turbine steam path engineering for operations and maintenance staff (first edition). Richmond Hill, Ontario: Canada: 236-239, 1996.

[10] Madhusudana, C.V., Thermal conductance of cylindrical joints. International Journal of Heat and Mass Transfer, 42: 1273-1287, 1999.

[11] Oden, J.T. \& Martins, J.A., Models and computational methods for dynamic friction phenomena. Computer methods in applied mechanics and engineering, 52: 527-634, 1985.

[12] Rajput, R.K., Heat and Mass Transfer, S. Chard \& Company Ltd: 30-40, 2006.

[13] Dimarogonas, A.D. \& Syrimbeis, N.B., Thermal signatures of vibrating rectangular plates. Journal of Sound and Vibration, 157(3): 467-476, 1992.

[14] Beards, C.F., Structural vibration: Analysis and damping, London: Elsevier Science: 200, 2003.

[15] Miller, S.F. \& Shih, A.J., Thermo-Mechanical Finite Element Modeling of the Friction Drilling Process. Journal of manufacturing science and engineering, 129: 531-538, 2007.

[16] Booysen, C., Heyns, P.S., Hindley, M.P. \& Scheepers, R., Fatigue life assessment of a low pressure steam turbine blade during transient resonant conditions using a probabilistic approach. International Journal of Fatigue, 73: 17-26, 2015.

[17] Koh, K.H., Griffin, J., Filppi, S. \& Akay, A., Characterization of turbine blade friction dampers. Proc. of ASME Turbo Expo 2005; Power for Land, Sea, and Air: Viena, Austria, 127: 856-862, 2005. 